\title{
SEMILINEAR ELLIPTIC EQUATIONS AND SYSTEMS WITH DIFFUSE MEASURES
}

\author{
LUIGI ORSINA AND AUGUSTO C. PONCE
}

A Lucio

"Tu se' lo mio maestro e 'l mio autore" (Dante, Inferno, I, 85)

\begin{abstract}
We study the equation $-\Delta u+g(x, u)=\mu$, where $g(\cdot, s)$ is finite outside sets of zero $H^{1}$-capacity, $\forall s \in \mathbb{R}$, and $\mu$ is a diffuse measure. As an application, we provide a positive answer to a question of Lucio Boccardo concerning existence of solutions of an elliptic system with absorption.
\end{abstract}

\section{CONTENTS}

1. Introduction and main results

2. Characterization of quasi- $L^{1}$ functions 4

$\begin{array}{ll}3 . & \text { Properties of equidiffuse sequences }\end{array}$

4. Stability of solutions of (1.4) 8

5. A variant of the method of sub and supersolutions 11

6. Proof of Theorem $1.2 \quad 12$

7. Proof of Theorem $1.1 \quad 13$

8. Proof of Theorem $1.3 \quad 15$

9. Study of a linear problem 16

Appendix A. A counterpart of the Hopf lemma for weak supersolutions 19

Appendix B. Failure of the method of sub and supersolutions 23

$\begin{array}{ll}\text { Acknowledgments } & 24\end{array}$

$\begin{array}{ll}\text { References } & 25\end{array}$

\section{INTRODUCTION AND MAIN RESULTS}

Let $\Omega \subset \mathbb{R}^{N}, N \geq 2$, be a smooth bounded domain. The original motivation of this work was a question of L. Boccardo concerning the existence of solutions of the system

$$
\left\{\begin{array}{rlrl}
-\Delta u+u^{p_{1}} v^{q_{1}} & =f_{1} & & \text { in } \Omega, \\
-\Delta v+u^{p_{2}} v^{q_{2}}=f_{2} & & \text { in } \Omega, \\
u=v & =0 & & \text { on } \partial \Omega,
\end{array}\right.
$$

where $f_{1}, f_{2}$ are given nonnegative functions in $L^{1}(\Omega)$ and $p_{i}, q_{i}>0$ for $i=1,2$. We prove in this paper that (1.1) always has a solution; see Theorem 1.1 below.

Date: September 17, 2008. 
More generally, we show that (1.1) still has a solution if $f_{1}, f_{2}$ are not necessarily $L^{1}$-functions, but diffuse measures.

We recall that a finite measure $\mu$ in $\Omega$ is diffuse if for every Borel set $E \subset \Omega$

$$
\operatorname{cap}(E)=0 \quad \Longrightarrow \quad|\mu|(E)=0,
$$

where "cap" denotes the Newtonian $H^{1}$-capacity. According to a result of BoccardoGallouët-Orsina [2], $\mu$ is diffuse if, and only if, $\mu \in L^{1}(\Omega)+H^{-1}(\Omega)$, i.e.

$$
\mu=f-\Delta u \text { in } \mathcal{D}^{\prime}(\Omega),
$$

for some $f \in L^{1}(\Omega)$ and $u \in H_{0}^{1}(\Omega)$.

One of our main results is the

THEOREM 1.1. Assume that $\mu_{1}, \mu_{2}$ are diffuse measures in $\Omega$ and $g_{1}, g_{2} \in C(\mathbb{R} \times \mathbb{R})$ satisfy

$\left(a_{1}\right)$ for every $t \in \mathbb{R}, g_{1}(\cdot, t)$ is nondecreasing and $g_{1}(0, t)=0$;

$\left(a_{2}\right)$ for every $s \in \mathbb{R}, g_{2}(s, \cdot)$ is nondecreasing and $g_{2}(s, 0)=0$.

Then, the system

$$
\left\{\begin{aligned}
-\Delta u+g_{1}(u, v) & =\mu_{1} & & \text { in } \Omega, \\
-\Delta v+g_{2}(u, v) & =\mu_{2} & & \text { in } \Omega, \\
u=v & =0 & & \text { on } \partial \Omega,
\end{aligned}\right.
$$

has a solution $(u, v) \in L^{1}(\Omega) \times L^{1}(\Omega)$.

Our proof of Theorem 1.1 is based on Schauder's fixed point theorem. Some important tools are the notions of "quasi- $L^{1}$ functions" and "equidiffuse sequences" (see Sections 2 and 3 below) as well as the following result concerning the existence of solutions of the scalar equation

$$
\left\{\begin{aligned}
-\Delta u+g(x, u) & =\mu & & \text { in } \Omega, \\
u & =0 & & \text { on } \partial \Omega .
\end{aligned}\right.
$$

TheOREM 1.2. Let $g: \Omega \times \mathbb{R} \rightarrow \mathbb{R}$ be a Carathéodory function such that

$$
g(x, s) s \geq 0 \quad \text { for a.e. } x \in \Omega, \forall s \in \mathbb{R}
$$

and

$$
\sup _{|s| \leq t}|g(x, s)| \leq G_{t}(x)+H_{t}(x) \quad \text { for a.e. } x \in \Omega, \forall t>0,
$$

where $G_{t}: \Omega \rightarrow \mathbb{R}$ is quasifinite and $H_{t} \in L^{1}(\Omega)$. If $\mu$ is a diffuse measure in $\Omega$, then there exist a smallest and a largest solution of (1.4).

We recall that a measurable function $G: \Omega \rightarrow \mathbb{R}$ is quasifinite if for every $\varepsilon>0$ and every $K \subset \Omega$ compact there exist $M>0$ and an open set $\omega \subset \Omega$ such that $\operatorname{cap}(\omega)<\varepsilon$ and $|G| \leq M$ a.e. on $K \backslash \omega$ (see [13]).

The study of the equation (1.4) with datum $\mu$ in $L^{1}(\Omega)$ was initiated by BrezisStrauss [7]. Later, Gallouët-Morel [11] studied the existence of solutions of (1.4) when $\mu \in L^{1}(\Omega)$ and

$$
\sup _{|s| \leq t}|g(\cdot, s)| \in L^{1}(\Omega) \quad \forall t>0
$$

in other words, when (1.6) holds with $G_{t} \equiv 0$. 
REMARK 1.1. Quasifinite functions need not belong to $L^{1}(\Omega)$; for example, $1 /\|x\|^{N}$ is quasifinite in $B_{1}$ but $1 /\|x\|^{N} \notin L^{1}\left(B_{1}\right)$. Conversely, $L^{1}$-functions need not be quasifinite; for instance, $1 /\left|x_{1}\right|^{\alpha} \in L^{1}\left(B_{1}\right)$ for any $0<\alpha<1$ but this function is not quasifinite since the set $\left[x_{1}=0\right] \cap B_{1}$ has positive $H^{1}$-capacity. This explains the presence of both $G_{t}$ and $H_{t}$ in (1.6). The possibility of allowing the term $G_{t}$ is needed in the proof of Theorem 1.1.

It follows from Theorem 1.2 that (1.4) always has a solution with

$$
g(x, s)=\frac{a(s)}{\|x\|^{\alpha}} \quad \forall(x, s) \in \mathbb{R}^{N} \times \mathbb{R},
$$

for every $\alpha>0$ and $a \in C(\mathbb{R})$, where $a(s) s \geq 0, \forall s \in \mathbb{R}$. On the other hand, the function $g$ given by

$$
g(x, s)=\frac{s}{\left|x_{1}\right|^{\alpha}} \quad \forall(x, s) \in \mathbb{R}^{N} \times \mathbb{R}
$$

does not satisfy condition (1.6) if $\alpha \geq 1$. Actually, for such $g$ we prove the following TheOREM 1.3. Let $1 \leq \alpha<2$. If $u \in L^{1}\left(B_{1}\right)$ is such that $u /\left|x_{1}\right|^{\alpha} \in L^{1}\left(B_{1}\right)$ and

$$
-\int_{B_{1}} u \Delta \zeta+\int_{B_{1}} \frac{u}{\left|x_{1}\right|^{\alpha}} \zeta \geq 0 \quad \forall \zeta \in C_{0}^{2}\left(\bar{B}_{1}\right), \zeta \geq 0 \text { in } B_{1},
$$

then

$$
u=0 \quad \text { a.e. in } B_{1} \text {. }
$$

Hence, according to Theorem 1.3 the equation

$$
\left\{\begin{aligned}
-\Delta u+\frac{u}{\left|x_{1}\right|^{\alpha}}=\mu & \text { in } B_{1} \\
u=0 & \text { on } \partial B_{1}
\end{aligned}\right.
$$

has no solution if $1 \leq \alpha<2$ and $\mu$ is a nonnegative measure, unless $\mu=0$. If $\alpha \geq 2$, then we show in Section 9 below that there do exist functions $u \in L^{1}(\Omega)$ satisfying (1.9) for every $\mu \in L^{1}\left(B_{1}\right)$, in the sense that $u /\left|x_{1}\right|^{\alpha} \in L^{1}\left(B_{1}\right)$ and

$$
-\int_{B_{1}} u \Delta \zeta+\int_{B_{1}} \frac{u}{\left|x_{1}\right|^{\alpha}} \zeta=\int_{B_{1}} \zeta d \mu \quad \forall \zeta \in C_{0}^{2}\left(\bar{B}_{1}\right) .
$$

In [8], Dal Maso-Mosco studied the question of existence and uniqueness of solutions for problems of the form

$$
\left\{\begin{aligned}
-\Delta u+\nu u=f & & \text { in } \Omega, \\
u=0 & & \text { on } \partial \Omega,
\end{aligned}\right.
$$

where $\nu$ is a nonnegative diffuse Borel measure (possibly with infinite mass) and $f \in L^{2}(\Omega)$. Given $\alpha \geq 1$ and $\Omega=B_{1}$, take

$$
\nu_{\alpha}=\frac{1}{\left|x_{1}\right|^{\alpha}} d x \quad \text { and } \quad X_{\alpha}=H_{0}^{1}\left(B_{1}\right) \cap L^{2}\left(B_{1} ; \nu_{\alpha}\right) .
$$

It follows from [8] that for every $f \in L^{2}\left(B_{1}\right)$ there exists a unique $u \in X_{\alpha}$ such that

$$
\int_{B_{1}} \nabla u \cdot \nabla v+\int_{B_{1}} \frac{u}{\left|x_{1}\right|^{\alpha}} v=\int_{B_{1}} f v \quad \forall v \in X_{\alpha} .
$$


We point out that their result does not contradict Theorem 1.3 above. In fact, it follows from Proposition 9.1 below that (1.13) holds for some $u \in X_{\alpha}$ if, and only if,

$$
\int_{B_{1}} \nabla u \cdot \nabla \varphi+\int_{B_{1}} \frac{u}{\left|x_{1}\right|^{\alpha}} \varphi=\int_{B_{1}} f \varphi \quad \forall \varphi \in C_{0}^{\infty}\left(B_{1}^{+} \cup B_{1}^{-}\right)
$$

where

$$
B_{1}^{+}=\left\{x \in B_{1} ; x_{1}>0\right\} \quad \text { and } \quad B_{1}^{-}=\left\{x \in B_{1} ; x_{1}<0\right\} .
$$

Moreover, for every function $u \in X_{\alpha}$,

$$
u \in H_{0}^{1}\left(B_{1}^{+}\right) \cup H_{0}^{1}\left(B_{1}^{-}\right)
$$

see Lemma 9.1 below. Hence, for every $\alpha \geq 1$, to find a solution of (1.11) in $B_{1}$ in the sense of [8] amounts to solve two independent Dirichlet problems on $B_{1}^{+}$and $B_{1}^{-}$, for which we know there is a solution. Indeed, it suffices to apply Theorem 1.2 in $B_{1}^{+}$and $B_{1}^{-}$with $G_{t}(x)=t /\left|x_{1}\right|^{\alpha}$ and $F_{t}(x)=0$. Note that in this case the parameter $\alpha$ plays no role whatsoever. The fact that the solution $u$ obtained this way satisfies (1.15) when $f \in L^{2}\left(B_{1}\right)$ follows from standard elliptic estimates (see $[8,14])$.

\section{Characterization OF QUASI- $L^{1}$ FUnCTIOnS}

In this section we discuss the concept of "quasi- $L^{1}$ functions" presented below:

Definition 2.1. A measurable function $F: \Omega \rightarrow \mathbb{R}$ is quasi- $L^{1}$ if for every $\varepsilon>0$ and every $K \subset \Omega$ compact there exists an open set $\omega \subset \Omega$ such that cap $(\omega)<\varepsilon$ and $F \in L^{1}(K \backslash \omega)$.

The motivation of Definition 2.1 comes from the well-known notion of quasicontinuity, which we recall below:

Definition 2.2. A measurable function $G: \Omega \rightarrow \mathbb{R}$ is quasicontinuous if for every $\varepsilon>0$ there exists an open set $\omega \subset \Omega$ such that cap $(\omega)<\varepsilon$ and $G$ is continuous on $\Omega \backslash \omega$.

For example, if $u \in L^{1}(\Omega)$ is such that

$$
\left|\int_{\Omega} u \Delta \varphi\right| \leq C\|\varphi\|_{L^{\infty}} \quad \forall \varphi \in C_{0}^{\infty}(\Omega),
$$

then by the Riesz Representation Theorem $\Delta u$ is a finite measure in $\Omega$; hence, there exists a quasicontinuous function $G: \Omega \rightarrow \mathbb{R}$ such that $G=u$ a.e. (see e.g. $\left[4\right.$, Lemma 1]). In particular, $u$ is quasi- $L^{1}$.

Clearly,

$$
F \text { quasicontinuous } \Longrightarrow F \text { quasifinite } \Longrightarrow F \text { quasi- } L^{1} \text {. }
$$

Simple examples show that the reverse implications are not true. Note in addition that

$\left(A_{1}\right)$ If $F_{1}, F_{2}: \Omega \rightarrow \mathbb{R}$ are measurable functions such that $F_{1}=F_{2}$ a.e. and if $F_{1}$ is quasi- $L^{1}$, then $F_{2}$ is also quasi- $L^{1}$;

$\left(A_{2}\right)$ If $F$ is quasi- $L^{1}$ in $\Omega$ and $u: \Omega \rightarrow \mathbb{R}$ is a measurable function such that $|u| \leq F$ a.e., then $u$ is also quasi- $L^{1}$. 
Similar properties also hold for quasifinite functions, but their counterparts for quasicontinuous functions are false.

We prove the following characterization of quasi- $L^{1}$ functions:

Proposition 2.1. A measurable function $F$ is quasi- $L^{1}$ in $\Omega$ if, and only if, there exist $G$ quasifinite in $\Omega$ and $H \in L^{1}(\Omega)$ such that

$$
|F| \leq G+H \quad \text { a.e. }
$$

Proof. The implication " $\Leftarrow$ " is clear since $G+H$ is quasi- $L^{1}$ by $(2.1)$, and so $F$ is quasi- $L^{1}$ by $\left(A_{2}\right)$. Conversely, assume that $F: \Omega \rightarrow \mathbb{R}$ is a quasi- $L^{1}$ function. We split the proof in two steps:

Step 1. Assume in addition that $F$ has compact support in $\Omega$. Then, given $\varepsilon>0$, (2.2) holds for some $G$ quasifinite and $H \in L^{1}(\Omega)$ such that $\|H\|_{L^{1}}<\varepsilon$.

Since $F$ has compact support, for each $k \geq 1$ one can find an open set $\omega_{k} \subset \subset \Omega$ with

$$
\operatorname{cap}\left(\omega_{k}\right)<\frac{1}{2^{k}} \quad \text { and } \quad F \in L^{1}\left(\Omega \backslash \omega_{k}\right) .
$$

We can assume that the sequence $\left(\omega_{k}\right)$ is non-increasing. For otherwise, we could take $\tilde{\omega}_{k}=\bigcup_{j=k+1}^{+\infty} \omega_{j}, \forall j \geq 1$; then, $\left(\tilde{\omega}_{k}\right)$ is non-increasing and still satisfies (2.3). For every $k \geq 1$, choose $M_{k}>0$ sufficiently large so that

For every $x \in \Omega$, let

$$
\int_{\substack{\Omega \backslash \omega_{k} \\\left[|F|>M_{k}\right]}}|F|<\frac{\varepsilon}{2^{k+1}} .
$$

$$
\begin{aligned}
& G(x)=\sum_{k \geq 1} M_{k} \chi_{\omega_{k-1} \backslash \omega_{k}}(x), \\
& H(x)=\sum_{k \geq 1}\left(|F(x)|-M_{k}\right)^{+} \chi_{\omega_{k-1} \backslash \omega_{k}}(x),
\end{aligned}
$$

where $\omega_{0}:=\Omega$. Then,

$$
|F| \leq G+H \text { a.e., } \quad H \in L^{1}(\Omega) \quad \text { and } \quad\|H\|_{L^{1}} \leq \varepsilon .
$$

Since $G$ is uniformly bounded on $\Omega \backslash \omega_{j}$ for every $j \geq 1$, it follows that $G$ is quasifinite. This concludes the proof of Step 1.

Step 2. Proof of Theorem 2.1 completed.

Write $\Omega=\bigcup_{n \geq 1} \Omega_{n}$ as an increasing union of open sets $\Omega_{n} \subset \subset \Omega$, and define $\Omega_{0}=\emptyset$. Applying the previous step to $F \chi_{\Omega_{n}}$, one finds a quasifinite function $G_{n}: \Omega \rightarrow \mathbb{R}$ and $H_{n} \in L^{1}(\Omega)$ supported in $\bar{\Omega}_{n}$ such that

$$
|F| \leq G_{n}+H_{n} \text { a.e. in } \Omega_{n} \text { and }\left\|H_{n}\right\|_{L^{1}} \leq \frac{1}{2^{n}} .
$$

The functions

$$
G(x)=\sum_{n \geq 1} G_{n}(x) \chi_{\Omega_{n-1} \backslash \Omega_{n}}(x) \quad \text { and } \quad H(x)=\sum_{n \geq 1} H_{n}(x) \quad \forall x \in \Omega
$$

satisfy all the required properties. The proof is complete.

We warn the reader of the following facts: 
$\left(A_{3}\right)$ If $G$ is quasifinite, then $G$ need not belong to $L^{1}(\Omega)$. Indeed, $G(x)=\frac{1}{\|x\|^{\alpha}}$ is quasifinite in the ball $B_{1} \subset \mathbb{R}^{N}$ for every $\alpha>0$, but $G \in L^{1}\left(B_{1}\right)$ if, and only if, $\alpha<N$.

$\left(A_{4}\right)$ If $G_{1}$ and $G_{2}$ are quasifinite functions in $\Omega$ such that $G_{1}=G_{2}$ a.e., then it need not be true that $\int_{\Omega} G_{1} d \mu$ and $\int_{\Omega} G_{2} d \mu$ coincide for a given diffuse measure $\mu$, even if $G_{1}, G_{2}$ are bounded functions; indeed, let $S$ be a segment in $\mathbb{R}^{2}, G_{1}=0, G_{2}=\chi_{S}$, and $\mu$ be the restriction of the 1-dimensional Hausdorff measure to $S$.

\section{Properties of equidiffuse sequences}

We denote by $\mathcal{M}(\Omega)$ the space of finite measures $\mu$ in $\Omega$ equipped with the norm

$$
\|\mu\|_{\mathcal{M}}:=|\mu|(\Omega)=\int_{\Omega}|\mu|
$$

We recall the (see [6])

DEFINITION 3.1. Given a sequence of finite measures $\left(\mu_{n}\right)$ in $\Omega$, we say that $\left(\mu_{n}\right)$ is equidiffuse if

(i) $\left(\mu_{n}\right)$ is bounded in $\mathcal{M}(\Omega)$;

(ii) Given $\varepsilon>0$, there exists $\delta>0$ such that for every Borel set $E \subset \Omega$

$$
\operatorname{cap}(E)<\delta \quad \Longrightarrow \quad\left|\mu_{n}\right|(E)<\varepsilon \quad \forall n \geq 1 \text {. }
$$

If a sequence $\left(\mu_{n}\right)$ is equidiffuse, then each measure $\mu_{n}$ is diffuse in view of the following

LEMMA 3.1. Let $\mu$ be a finite measure in $\Omega$. Then, $\mu$ is diffuse if, and only if,

$$
\lim _{\operatorname{cap}(E) \rightarrow 0} \mu(E)=0 .
$$

Proof. $(\Leftarrow)$ Given $\varepsilon>0$, let $\delta>0$ be such that

$$
\operatorname{cap}(E)<\delta \quad \Longrightarrow \quad|\mu|(E)<\varepsilon \text {. }
$$

If $E_{0} \subset \Omega$ is a Borel set such that cap $\left(E_{0}\right)=0$, then $|\mu|\left(E_{0}\right)<\varepsilon, \forall \varepsilon>0$. Thus, $|\mu|\left(E_{0}\right)=0$ and $\mu$ is diffuse.

$(\Rightarrow)$ We may assume that $\mu \geq 0$; the case of signed measures then follows by applying the conclusion to $|\mu|$. Reasoning by contradiction, suppose that there exist $\varepsilon_{0}>0$ and a sequence $\left(E_{n}\right)$ of Borel subsets of $\Omega$ such that cap $\left(E_{n}\right)$ tends to zero but $\mu\left(E_{n}\right)>\varepsilon_{0}$ for every $n \geq 1$. If the sequence $\left(E_{n}\right)$ is decreasing, then the set $E=\bigcap_{n=1}^{+\infty} E_{n}$ has zero capacity and is such that $\mu(E) \geq \varepsilon_{0}$, a contradiction. If the sequence $\left(E_{n}\right)$ is not decreasing, consider a subsequence $\left(E_{n_{j}}\right)$ such that cap $\left(E_{n_{j}}\right)<$ $2^{-j}$ for every $j \geq 1$. Then, the sequence $\left(F_{k}\right)$ given by $F_{k}=\bigcup_{j=k+1}^{+\infty} E_{n_{j}}$ is decreasing, with capacity smaller than $2^{-k}$, and is such that $\mu\left(F_{k}\right)>\varepsilon_{0}$. The conclusion then follows as before.

A first example of an equidiffuse sequence $\left(\mu_{n}\right)$ is the

$\left(B_{1}\right) \mu_{n}=\mu$, where $\mu$ is a given diffuse measure.

This follows from Lemma 3.1 above. Other examples are

$\left(B_{2}\right) \mu_{n}=\rho_{n} * \mu$, where $\mu$ is diffuse and $\left(\rho_{n}\right)$ is a sequence of mollifiers;

$\left(B_{3}\right) \mu_{n}=f_{n}$, where $\left(f_{n}\right)$ is an equi-integrable sequence in $L^{1}(\Omega)$;

$\left(B_{4}\right) \mu_{n}=\Delta u_{n}$, where $\left(u_{n}\right)$ is a bounded sequence in $H_{0}^{1}(\Omega)$. 
Clearly, sums of equidiffuse sequences are still equidiffuse. In view of $\left(B_{3}\right)-\left(B_{4}\right)$, one deduces that if $\left(\mu_{n}\right)$ is a bounded sequence of measures such that

$$
\mu_{n}=f_{n}-\Delta u_{n} \quad \text { in } \mathcal{D}^{\prime}(\Omega)
$$

where $f_{n}$ and $u_{n}$ are as above, then $\left(\mu_{n}\right)$ is equidiffuse. It would be interesting to have a characterization of equidiffuse sequences in the spirit of the BoccardoGallouët-Orsina decomposition (1.2):

Open Problem 1. Let $\left(\mu_{n}\right)$ be an equidiffuse sequence converging weakly* in $\Omega$. Can one find $\left(f_{n}\right) \subset L^{1}(\Omega)$ and $\left(u_{n}\right) \subset H_{0}^{1}(\Omega)$ such that

$\left(b_{1}\right) \mu_{n}=f_{n}-\Delta u_{n}$ in $\mathcal{D}^{\prime}(\Omega)$;

$\left(b_{2}\right)\left(f_{n}\right)$ converges strongly in $L^{1}(\Omega)$;

$\left(b_{3}\right)\left(u_{n}\right)$ is bounded in $H_{0}^{1}(\Omega)$ ?

A connection between Definitions 2.1 and 3.1 is provided by the following

LEMMA 3.2. Let $\left(f_{n}\right)$ be a sequence in $L^{1}(\Omega)$ such that

$\left(c_{1}\right) f_{n} \rightarrow f$ a.e.;

$\left(c_{2}\right)\left|f_{n}\right| \leq F$ a.e., $\forall n \geq 1$, for some quasi- $L^{1}$ function $F$ in $\Omega$;

$\left(c_{3}\right)\left(f_{n}\right)$ is equidiffuse.

Then,

$$
f_{n} \rightarrow f \quad \text { in } L^{1}\left(\Omega ; \rho_{0} d x\right),
$$

where $\rho_{0}(x)=\operatorname{dist}(x, \partial \Omega), \forall x \in \Omega$.

A variant of this result was established by Lin-Ponce-Yang [13]. If $F$ is a (genuine) $L^{1}$-function in $\Omega$, then Lemma 3.2 just follows from Lebesgue's dominated convergence theorem (in which case $\left(c_{3}\right)$ is not needed). For general quasi- $L^{1}$ functions $F$, the conclusion (3.4) need not be true if $\left(c_{3}\right)$ fails.

Proof. Replacing $f_{n}$ by $f_{n}-f$ if necessary, we may assume that

$$
f_{n} \rightarrow 0 \text { a.e. }
$$

For every open set $A \subset \subset \Omega$, we show that

$$
f_{n} \rightarrow 0 \text { in } L^{1}(A) \text {. }
$$

By $\left(c_{3}\right)$, for every $\varepsilon>0$ there exists $\delta>0$ such that

$$
\operatorname{cap}(E)<\delta \quad \Longrightarrow \quad \int_{E}\left|f_{n}\right|<\varepsilon \quad \forall n \geq 1 .
$$

Since $F$ is quasi- $L^{1}$, one finds an open set $\omega \subset \Omega$ such that $\operatorname{cap}(\omega)<\delta$ and $F \in L^{1}(\bar{A} \backslash \omega)$. Thus, by $\left(c_{1}\right),\left(c_{2}\right)$, and dominated convergence,

$$
f_{n} \chi_{A \backslash \omega} \rightarrow 0 \text { in } L^{1}(A)
$$

Moreover, since we have $\operatorname{cap}(\omega)<\delta$, it follows from (3.7) that

$$
\int_{\omega}\left|f_{n}\right|<\varepsilon \quad \forall n \geq 1 \text {. }
$$

Thus,

$$
\limsup _{n \rightarrow \infty} \int_{A}\left|f_{n}\right| \leq \varepsilon
$$


Since $\varepsilon>0$ is arbitrary, we deduce that

$$
\lim _{n \rightarrow \infty} \int_{A}\left|f_{n}\right|=0
$$

This establishes (3.6) for every $A \subset \subset \Omega$. Since $\left(f_{n}\right)$ is bounded in $L^{1}(\Omega),(3.4)$ follows.

\section{Stability of SOlutions of (1.4)}

We recall that a function $u \in L^{1}(\Omega)$ is a solution of

$$
\left\{\begin{aligned}
-\Delta u=\mu & \text { in } \Omega, \\
u=0 & \text { on } \partial \Omega,
\end{aligned}\right.
$$

for a given finite measure $\mu$ in $\Omega$ if

$$
-\int_{\Omega} u \Delta \zeta=\int_{\Omega} \zeta d \mu \quad \forall \zeta \in C_{0}^{2}(\bar{\Omega}),
$$

where

$$
C_{0}^{2}(\bar{\Omega})=\left\{\zeta \in C^{2}(\bar{\Omega}) ; \zeta=0 \text { on } \partial \Omega\right\} .
$$

We say that $u \in L^{1}(\Omega)$ satisfies

$$
\left\{\begin{aligned}
-\Delta u+g(x, u) & =\mu & & \text { in } \Omega, \\
u & =0 & & \text { on } \partial \Omega,
\end{aligned}\right.
$$

where $g: \Omega \times \mathbb{R} \rightarrow \mathbb{R}$ is a Carathéodory function, if $g(\cdot, u) \in L^{1}(\Omega)$ and

$$
-\int_{\Omega} u \Delta \zeta+\int_{\Omega} g(x, u) \zeta=\int_{\Omega} \zeta d \mu \quad \forall \zeta \in C_{0}^{2}(\bar{\Omega}) .
$$

The main result of this section is the

Proposition 4.1. Let $g_{n}: \Omega \times \mathbb{R} \rightarrow \mathbb{R}$ be a sequence of Carathéodory functions such that

(i) $g_{n}(x, s) s \geq 0$ for a.e. $x \in \Omega, \forall s \in \mathbb{R}$;

(ii) $g_{n}\left(\cdot, s_{n}\right) \rightarrow g(\cdot, s)$ a.e. whenever $s_{n} \rightarrow s$ in $\mathbb{R}$;

(iii) for every $t \in \mathbb{R}$, there exists a quasi- $L^{1}$ function $F_{t}$ in $\Omega$ such that

$$
\sup _{|s| \leq t}\left|g_{n}(x, s)\right| \leq F_{t}(x) \quad \text { for a.e. } x \in \Omega, \quad \forall n \geq 1 .
$$

Given a diffuse measure $\mu$ in $\Omega$, assume that

$$
\left\{\begin{aligned}
-\Delta u_{n}+g_{n}\left(x, u_{n}\right) & =\mu & & \text { in } \Omega, \\
u_{n} & =0 & & \text { on } \partial \Omega .
\end{aligned}\right.
$$

has a solution $u_{n}, \forall n \geq 1$. Then, up to subsequences, $u_{n}$ converges strongly in $L^{1}(\Omega)$ to a solution $u$ of $(4.2)$.

In order to prove Proposition 4.1, we first recall some known results. We start with the uniform estimates for (4.1) (see [17]):

LEMMA 4.1. Every solution $u$ of (4.1) belongs to $W_{0}^{1, q}(\Omega)$, for $1 \leq q<\frac{N}{N-1}$, and

$$
\|u\|_{W_{0}^{1, q}} \leq C_{q}\|\mu\|_{\mathcal{M}} \text {. }
$$

The analog of Lemma 4.1 for the semilinear problem (4.2) is the following (see [3, Proposition B.3]) 
Lemma 4.2. Let $g: \Omega \times \mathbb{R} \rightarrow \mathbb{R}$ be a Carathéodory function such that

$$
g(x, s) s \geq 0 \quad \text { for a.e. } x \in \Omega, \forall s \in \mathbb{R} .
$$

Then, every solution $u$ of (4.2) satisfies

$$
\|g(\cdot, u)\|_{L^{1}} \leq\|\mu\|_{\mathcal{M}} .
$$

In particular, (4.4) holds.

The notion of (weak) sub and supersolutions of (4.2) we consider in this paper is given below:

DeFinition 4.1. A subsolution of (4.2) is a function $\underline{u} \in L^{1}(\Omega)$ such that $g(\cdot, \underline{u}) \in$ $L^{1}(\Omega)$ and

$$
-\int_{\Omega} \underline{u} \Delta \zeta+\int_{\Omega} g(x, \underline{u}) \zeta \leq \int_{\Omega} \zeta d \mu \quad \forall \zeta \in C_{0}^{2}(\bar{\Omega}), \zeta \geq 0 \text { in } \Omega .
$$

Analogously, a supersolution of (4.2) is a function $\bar{u} \in L^{1}(\Omega)$ such that $g(\cdot, \bar{u}) \in$ $L^{1}(\Omega)$ and

$$
-\int_{\Omega} \bar{u} \Delta \zeta+\int_{\Omega} g(x, \bar{u}) \zeta \geq \int_{\Omega} \zeta d \mu \quad \forall \zeta \in C_{0}^{2}(\bar{\Omega}), \zeta \geq 0 \text { in } \Omega .
$$

We show that if $g$ satisfies (4.5), then sub and supersolutions of (4.2) have "bounds" from above and from below:

Proposition 4.2. Let $\mu$ be a finite measure in $\Omega$, and let $\underline{U}$ and $\bar{U}$ be the (unique) solutions of

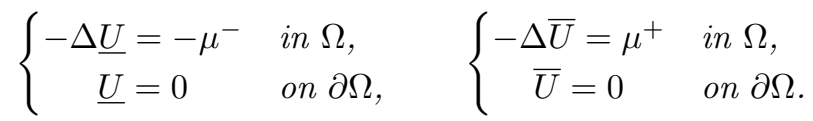

If (4.5) holds, then any subsolution $\underline{w}$ of (4.2) satisfies $\underline{w} \leq \bar{U}$ and any supersolution $\bar{w}$ of (4.2) satisfies $\underline{U} \leq \bar{w}$.

We present a proof of Proposition 4.2 based on the following version of Kato's inequality (see [3, Proposition B.5]):

Lemma 4.3. Let $w \in L^{1}(\Omega)$ and $f \in L^{1}\left(\Omega ; \rho_{0} d x\right)$ be such that

Then,

$$
-\int_{\Omega} w \Delta \zeta \leq \int_{\Omega} f \zeta \quad \forall \zeta \in C_{0}^{2}(\bar{\Omega}), \zeta \geq 0 \text { in } \Omega .
$$

$$
-\int_{\Omega} w^{+} \Delta \zeta \leq \int_{[w \geq 0]} f \zeta \quad \forall \zeta \in C_{0}^{2}(\bar{\Omega}), \zeta \geq 0 \text { in } \Omega .
$$

Proof of Proposition 4.2. If $\underline{w}$ is a subsolution of (4.2), then for every $\zeta \in C_{0}^{2}(\bar{\Omega})$ with $\zeta \geq 0$ in $\Omega$ we have

$$
\begin{aligned}
-\int_{\Omega}(\underline{w}-\bar{U}) \Delta \zeta & \leq-\int_{\Omega} g(x, \underline{w}) \zeta+\int_{\Omega} \zeta d \mu-\int_{\Omega} \zeta d \mu^{+} \\
& =-\int_{\Omega} g(x, \underline{w}) \zeta-\int_{\Omega} \zeta d \mu^{-} \\
& \leq-\int_{\Omega} g(x, \underline{w}) \zeta .
\end{aligned}
$$


Therefore, by Lemma 4.3,

$$
-\int_{\Omega}(\underline{w}-\bar{U})^{+} \Delta \zeta \leq-\int_{[\underline{w} \geq \bar{U}]} g(x, \underline{w}) \zeta \quad \forall \zeta \in C_{0}^{2}(\bar{\Omega}), \zeta \geq 0 \text { in } \Omega .
$$

Being $\bar{U} \geq 0$, we have $\underline{w} \geq 0$ on the set $[\underline{w} \geq \bar{U}]$; hence, by (4.5),

$$
g(x, \underline{w}) \geq 0 \quad \text { a.e. on }[\underline{w} \geq \bar{U}] .
$$

Combining (4.9)-(4.10), we deduce that

$$
-\int_{\Omega}(\underline{w}-\bar{U})^{+} \Delta \zeta \leq 0 \quad \forall \zeta \in C_{0}^{2}(\bar{\Omega}), \zeta \geq 0 \text { in } \Omega .
$$

Therefore, $(\underline{w}-\bar{U})^{+}=0$ a.e. in $\Omega$; equivalently, $\underline{w} \leq \bar{U}$ a.e. The inequality $\bar{w} \geq \underline{U}$ is proved in the same way.

The following result will be useful in the sequel

LEMMA 4.4. Let $\left(\mu_{n}\right)$ be a sequence of measures in $\Omega$ such that

$$
\mu_{n}=f_{n}-\Delta u_{n} \quad \text { in } \mathcal{D}^{\prime}(\Omega)
$$

for some $f_{n} \in L^{1}(\Omega)$ and $u_{n} \in W_{0}^{1,1}(\Omega)$ such that $f_{n} u_{n} \geq 0$ a.e., $\forall n \geq 1$. If $\left(\mu_{n}\right)$ is equidiffuse, then $\left(f_{n}\right)$ is also equidiffuse.

We refer the reader to [6] for a proof of Lemma 4.4; see also [15].

We now present the

Proof of Proposition 4.1. Using (i) and Lemma 4.2, we deduce that $\left(u_{n}\right)$ is bounded in $W_{0}^{1, q}(\Omega)$ for every $1 \leq q<\frac{N}{N-1}$. Therefore, by the Rellich-Kondrachov theorem, there exists a subsequence of $\left(u_{n}\right)$ (still denoted by $\left(u_{n}\right)$ ) which converges strongly in $L^{1}(\Omega)$, and almost everywhere, to a function $u$. In particular, by (ii)

$$
g_{n}\left(\cdot, u_{n}\right) \rightarrow g(\cdot, u) \quad \text { a.e. }
$$

We claim that $\left(g_{n}\left(\cdot, u_{n}\right)\right)$ also satisfies assumptions $\left(c_{2}\right)-\left(c_{3}\right)$ of Lemma 3.2. In fact, by Lemma 4.4, this sequence is equidiffuse. By Proposition 4.2, we know that

$$
\underline{U} \leq u_{n} \leq \bar{U} \quad \text { a.e., } \quad \forall n \geq 1 .
$$

Let $V=\max \{-\underline{U}, \bar{U}\} ; V$ is quasicontinuous since $\underline{U}$ and $\bar{U}$ are quasicontinuous. In particular, $V$ is quasifinite and $\left|u_{n}\right| \leq V$ a.e. Let

$$
W(x)=\sup _{n \in \mathbb{N}} \sup _{|s| \leq V(x)}\left|g_{n}(x, s)\right| \quad \text { for a.e. } x \in \Omega \text {. }
$$

Claim 1. $W$ is measurable.

Indeed, note that for each $n \geq 1$

$$
G_{n}(x, t)=\sup _{|s| \leq t}\left|g_{n}(x, s)\right| \quad \forall(x, t) \in \Omega \times \mathbb{R}
$$

is a Carathéodory function (see e.g. [10]). Since $V$ is measurable, it follows that $G_{n}(\cdot, V)$ is measurable as well. Hence, $W$ is also measurable being the supremum of countably many measurable functions.

Claim 2. $W$ is quasi- $L^{1}$. 
Given $K \subset \Omega$ and $\varepsilon>0$, let $M>0$ and $\omega_{1} \subset \Omega$ be an open set such that $\operatorname{cap}\left(\omega_{1}\right)<\varepsilon / 2$ and

$$
|V(x)| \leq M \quad \text { a.e. on } K \backslash \omega_{1} .
$$

Let $\omega_{2} \subset \Omega$ be such that cap $\left(\omega_{2}\right)<\varepsilon / 2$ and

$$
F_{M} \in L^{1}\left(K \backslash \omega_{2}\right) \text {. }
$$

Take $\omega_{0}=\omega_{1} \cup \omega_{2}$. Then, $\operatorname{cap}\left(\omega_{0}\right)<\varepsilon$ and

$$
0 \leq W(x) \leq \sup _{n \in \mathbb{N}} \sup _{|s| \leq M}\left|g_{n}(x, s)\right| \leq F_{M}(x) \quad \text { a.e. on } K \backslash \omega_{0} .
$$

Thus, the function $W$ belongs to $L^{1}\left(K \backslash \omega_{0}\right)$, and is therefore quasi- $L^{1}$. This establishes Claim 2.

Since $W$ is quasi- $L^{1}$,

$$
\left|g_{n}\left(\cdot, u_{n}\right)\right| \leq W \quad \text { a.e., } \quad \forall n \geq 1,
$$

and $\left(g_{n}\left(\cdot, u_{n}\right)\right)$ is equidiffuse, by Lemma 3.2 we have

$$
g_{n}\left(\cdot, u_{n}\right) \rightarrow g(\cdot, u) \quad \text { in } L^{1}\left(\Omega ; \rho_{0} d x\right) .
$$

Note that for every function $\zeta$ in $C_{0}^{2}(\bar{\Omega})$ there exists a constant $C>0$ such that $|\zeta| \leq C \rho_{0}$. Hence, the convergence of $\left(g_{n}\left(\cdot, u_{n}\right)\right)$ in $L^{1}\left(\Omega ; \rho_{0} d x\right)$ is enough in order to pass to the limit in the weak formulation of (4.3) and we get

$$
-\int_{\Omega} u \Delta \zeta+\int_{\Omega} g(x, u) \zeta=\int_{\Omega} \zeta d \mu \quad \forall \zeta \in C_{0}^{2}(\bar{\Omega}) .
$$

In view of the pointwise convergence (4.12), by Lemma 4.2 and Fatou's lemma we have $g(\cdot, u) \in L^{1}(\Omega)$. Thus, $u$ is a solution of $(4.2)$.

\section{A VARIANT OF THE METHOD OF SUB AND SUPERSOLUTIONS}

Thanks to the stability result in the previous section, we can now establish the following version of the method of sub and supersolutions for problem (1.4):

Proposition 5.1. Let $g: \Omega \times \mathbb{R} \rightarrow \mathbb{R}$ be a Carathéodory function satisfying (1.5)(1.6). Given a diffuse measure $\mu$ in $\Omega$, assume that (1.4) has sub and supersolutions $\underline{w} \leq \bar{w}$ a.e. Then, there exists a solution $u$ of (1.4) with $\underline{w} \leq u \leq \bar{w}$ a.e.

In Appendix B below, we show that the conclusion need not hold if the measure $\mu$ is not diffuse. The main difference between Proposition 5.1 and [16, Corollary 5.4] (whose conclusion is true for every finite measure $\mu$ ) is that in the statement above it need not be true that

$$
\underline{w} \leq v \leq \bar{w} \quad \Longrightarrow \quad g(\cdot, v) \in L^{1}(\Omega) .
$$

Proof. Let

$$
h(x)=|g(x, \underline{w}(x))|+|g(x, \bar{w}(x))|+1 ;
$$

then $h$ belongs to $L^{1}(\Omega)$. Let

$$
g_{n}(x, s)= \begin{cases}-n h(x) & \text { if } g(x, s) \leq-n h(x), \\ g(x, s) & \text { if }-n h(x)<g(x, s)<n h(x), \\ n h(x) & \text { if } g(x, s) \geq n h(x) .\end{cases}
$$

In particular,

$$
\left|g_{n}(\cdot, s)\right| \leq n h \quad \forall s \in \mathbb{R}, \quad \forall n \geq 1
$$


where $n h \in L^{1}(\Omega)$. Since

$$
g_{n}(x, \bar{w})=g(x, \bar{w}) \quad \text { and } \quad g_{n}(x, \underline{w})=g(x, \underline{w}),
$$

it follows that $\bar{w}$ and $\underline{w}$ still are sub and supersolutions of

$$
\left\{\begin{aligned}
-\Delta u+g_{n}(x, u) & =\mu & & \text { in } \Omega, \\
u & =0 & & \text { on } \partial \Omega .
\end{aligned}\right.
$$

Since, by (5.1),

$$
\underline{w} \leq v \leq \bar{w} \quad \Longrightarrow \quad g_{n}(\cdot, v) \in L^{1}(\Omega),
$$

applying [16, Corollary 5.4] we deduce that equation (5.2) has a solution $u_{n}$ such that

$$
\underline{w} \leq u_{n} \leq \bar{w} \quad \text { a.e. }
$$

Clearly, the sequence $\left(g_{n}\right)$ satisfies assumptions (i)-(ii) of Proposition 4.1. Observe now that, by construction, $\left|g_{n}\right| \leq|g|$ in $\Omega \times \mathbb{R}$; thus, by (1.6),

$$
\sup _{|s| \leq t}\left|g_{n}(x, s)\right| \leq G_{t}(x)+H_{t}(x) \quad \text { a.e. } \quad \forall t>0 .
$$

By Proposition 2.1, $G_{t}+H_{t}$ is quasi- $L^{1}$. It thus follows from Proposition 4.1 that (up to a subsequence) $\left(u_{n}\right)$ strongly converges in $L^{1}(\Omega)$ to a solution $u$ of

$$
\left\{\begin{aligned}
-\Delta u+g(x, u) & =\mu & & \text { in } \Omega, \\
u & =0 & & \text { on } \partial \Omega .
\end{aligned}\right.
$$

Furthermore, $\underline{w} \leq u \leq \bar{w}$ a.e. as desired.

\section{Proof of Theorem 1.2}

In order to apply the results of the previous section, we first need to show that for any given diffuse measure $\mu$ equation (1.4) does have sub and supersolutions associated to $\mu$ in the sense of Definition 4.1. This is established in our next

LEMMA 6.1. Let $g: \Omega \times \mathbb{R} \rightarrow \mathbb{R}$ be a Carathéodory function satisfying (1.5)-(1.6). Given a diffuse measure $\mu$ on $\Omega$, then equation (1.4) has sub and supersolutions $\underline{w} \leq \bar{w}$ a.e. such that any solution $u$ of (1.4) satisfies

$$
\underline{w} \leq u \leq \bar{w} \quad \text { a.e. }
$$

Proof. We first show the existence of $\bar{w}$. For this purpose, let

$$
g_{n}(x, s)=\left\{\begin{array}{cl}
g(x, s) & \text { if } g(x, s) \leq n, \\
n & \text { if } g(x, s)>n .
\end{array}\right.
$$

Since $g_{n}$ is bounded from above, by [16, Corollary 5.4] applied with sub and supersolutions $0 \leq \bar{U}$, the equation

$$
\left\{\begin{aligned}
-\Delta u+g_{n}(x, u) & =\mu^{+} & & \text {in } \Omega, \\
u & =0 & & \text { on } \partial \Omega,
\end{aligned}\right.
$$

has a largest solution $u_{n}$ in $[0, \bar{U}]$. Since

$$
g_{n-1}\left(x, u_{n}\right) \leq g_{n}\left(x, u_{n}\right) \quad \text { a.e. }
$$

$u_{n}$ is a subsolution for the problem solved by $u_{n-1}$. By Proposition 4.2 and the maximality of $u_{n-1}$, this implies that $u_{n-1} \geq u_{n}$ a.e. In other words, the sequence $\left(u_{n}\right)$ is non-increasing and bounded from below by 0 . Let $\bar{w}$ be the limit of $\left(u_{n}\right)$. 
By Proposition 4.1, $\bar{w}$ is a solution of (1.4) with datum $\mu^{+}$.

We claim that any solution $u$ of (1.4) satisfies

$$
u \leq \bar{w} \text { a.e. }
$$

Indeed, $u$ is a subsolution of (6.1). By maximality of $u_{n}$, we have

$$
u \leq u_{n} \quad \text { a.e., } \quad \forall n \geq 1 \text {. }
$$

As $n \rightarrow \infty$, we deduce (6.2). The existence of a subsolution $\underline{w}$ is established in a similar way using $-\mu^{-}$as datum.

Remark that in the proof $\bar{w}$ has been chosen as the largest solution of (1.4) with datum $\mu^{+}$and $\underline{w}$ as the smallest solution of (1.4) with datum $-\mu^{-}$.

Proof of Theorem 1.2. Applying Lemma 6.1 and Proposition 5.1, we deduce that (1.4) has a solution. We now show that (1.4) has a largest solution. Before proceeding, we first establish the following

Claim. Given two solutions $u_{1}$ and $u_{2}$ of (1.4), there exists a solution $u$ such that $u \geq \max \left\{u_{1}, u_{2}\right\}$.

By [16, Corollary 3.1], for any two solutions $u_{1}$ and $u_{2}$ of (1.4) the function $\max \left\{u_{1}, u_{2}\right\}$ is a subsolution of the same problem. Applying Proposition 5.1 with sub and supersolutions $\max \left\{u_{1}, u_{2}\right\} \leq \bar{w}$, one finds a solution $u$ such that

$$
\max \left\{u_{1}, u_{2}\right\} \leq u \leq \bar{w} \text { a.e. }
$$

This establishes the claim.

In order to prove the existence of the largest solution of (1.4), we follow the lines of [16]. Let

$$
A=\sup \left\{\int_{\Omega} u ; u \text { is a solution of (1.4) }\right\} .
$$

By Proposition 4.2, $\underline{U} \leq u \leq \bar{U}$ a.e. for every solution of (1.4); thus, since $\underline{U}$ and $\bar{U}$ are in $L^{1}(\Omega), A$ is finite. By the claim above, we can find a nondecreasing sequence $\left(u_{n}\right)$ of solutions of (1.4) such that

$$
\int_{\Omega} u_{n} \rightarrow A
$$

By monotone convergence, there exists $u_{0}$ in $L^{1}(\Omega)$, limit of $u_{n}$, such that

$$
\int_{\Omega} u_{0}=A
$$

Applying Proposition 4.1, we deduce that $u_{0}$ is a solution of (1.4). Hence, by the claim $u_{0}$ must be the largest solution of (1.4). The existence of the smallest solution is achieved in the same way.

\section{Proof of Theorem 1.1}

Let $\bar{U}$ and $\underline{U}$ be the solutions of

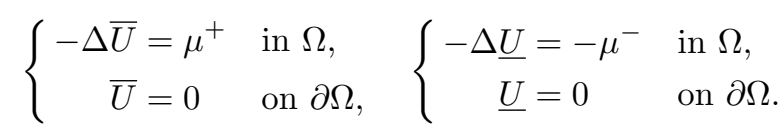


Similarly, let $\bar{V}$ and $\underline{V}$ be the solutions of the same problems with data $\nu^{+}$and $-\nu^{-}$. Define

$$
K_{\mu}=\left\{w \in L^{1}(\Omega) ; \underline{U} \leq w \leq \bar{U}\right\} \quad \text { and } \quad K_{\nu}=\left\{z \in L^{1}(\Omega) ; \underline{V} \leq z \leq \bar{V}\right\},
$$

so that both $K_{\mu}$ and $K_{\nu}$ are closed convex subsets of $L^{1}(\Omega)$. Since $\bar{U}, \underline{U}, \bar{V}$ and $\underline{V}$ are quasifinite, then any function in $K_{\mu}$ and $K_{\nu}$ is quasifinite. Given $z \in K_{\nu}$, consider

$$
h_{1}(x, s)=g_{1}(s, z(x)) \quad \forall(x, s) \in \Omega \times \mathbb{R} .
$$

Then, $h_{1}$ is a Carathéodory function which satisfies (1.5)-(1.6) (the latter holds since $g_{1}$ is continuous and $z$ is quasifinite). Therefore, by Theorem 1.2 there exists a solution $u$ of

$$
\left\{\begin{aligned}
-\Delta u+g_{1}(u, z(x)) & =\mu & & \text { in } \Omega, \\
u & =0 & & \text { on } \partial \Omega .
\end{aligned}\right.
$$

Since $h_{1}(x, \cdot)$ is nondecreasing, the solution of (7.1) is unique (see [3, Corollary B.1]). Given $w$ in $K_{\mu}$ define

$$
h_{2}(x, t)=g_{2}(w(x), t) \quad \forall(x, t) \in \Omega \times \mathbb{R} .
$$

In the same way as before, there exists a unique solution $v$ of

$$
\left\{\begin{aligned}
-\Delta v+g_{2}(w(x), v) & =\nu & & \text { in } \Omega, \\
v & =0 & & \text { on } \partial \Omega .
\end{aligned}\right.
$$

By Proposition 4.2, $u$ belongs to $K_{\mu}$ and $v$ belongs to $K_{\nu}$. Thus, the map

$$
\begin{aligned}
T: K_{\mu} \times K_{\nu} & \longrightarrow K_{\mu} \times K_{\nu} \\
(w, z) & \longmapsto(u, v)
\end{aligned}
$$

is well-defined. By Lemma 4.2, we have

$$
\|u\|_{W_{0}^{1, q}} \leq C\|\mu\|_{\mathcal{M}} \text { and } \quad\|v\|_{W_{0}^{1, q}} \leq C\|\nu\|_{\mathcal{M}}
$$

for every $1 \leq q<\frac{N}{N-1}$. Hence, by the Rellich-Kondrachov theorem, $T\left(K_{\mu} \times K_{\nu}\right)$ is bounded and relatively compact in $L^{1}(\Omega) \times L^{1}(\Omega)$.

We now show that $T$ is continuous. For this purpose, let $\left(z_{n}\right)$ be a sequence of functions in $K_{\nu}$ such that $z_{n} \rightarrow z$ in $L^{1}(\Omega)$. Let $u_{n}$ be the corresponding solutions of

$$
\left\{\begin{array}{rlrl}
-\Delta u_{n}+g_{1}\left(u_{n}, z_{n}(x)\right) & =\mu & & \text { in } \Omega, \\
u_{n}=0 & & \text { on } \partial \Omega .
\end{array}\right.
$$

By Proposition 4.1, there exists a subsequence $\left(u_{n_{k}}\right)$ such that $u_{n_{k}} \rightarrow u$ in $L^{1}(\Omega)$, where $u$ is the solution of

$$
\left\{\begin{aligned}
-\Delta u+g_{1}(u, z(x)) & =\mu & & \text { in } \Omega, \\
u & =0 & & \text { on } \partial \Omega .
\end{aligned}\right.
$$

By uniqueness of $u$, the whole sequence $\left(u_{n}\right)$ converges to $u$. Analogously, if the sequence $\left(w_{n}\right)$ in $K_{\mu}$ strongly converges in $L^{1}(\Omega)$ to $w$, then the sequence $\left(v_{n}\right)$ of solutions of

$$
\left\{\begin{aligned}
-\Delta v_{n}+g_{1}\left(w_{n}(x), v_{n}\right) & =\nu & & \text { in } \Omega, \\
v_{n} & =0 & & \text { on } \partial \Omega,
\end{aligned}\right.
$$


strongly converges in $L^{1}(\Omega)$ to the solution of

$$
\left\{\begin{aligned}
-\Delta v+g_{2}(w(x), v) & =\nu & & \text { in } \Omega, \\
v & =0 & & \text { on } \partial \Omega .
\end{aligned}\right.
$$

Hence, $T$ is continuous. Therefore, by Schauder's theorem, there exists a fixed point $(u, v)$ of $T$, that is, a solution of (1.3).

REMARK 7.1. We do not know whether Theorem 1.1 still holds if $\left(a_{1}\right)-\left(a_{2}\right)$ are replaced by the weaker assumptions:

$\left(\tilde{a}_{1}\right) g_{1}(\cdot, t) t \geq 0$ for every $t \in \mathbb{R}$;

$\left(\tilde{a}_{2}\right) g_{2}(s, \cdot) s \geq 0$ for every $s \in \mathbb{R}$.

Note that $\left(\tilde{a}_{1}\right)-\left(\tilde{a}_{2}\right)$ guarantee the existence of solutions of (7.1)-(7.2) (via Theorem 1.2), but in this case $u$ and $v$ need not be unique. One could define for example $T: K_{\mu} \times K_{\nu} \rightarrow K_{\mu} \times K_{\nu}$ as $T(w, z)=(\bar{u}, \bar{v})$, where $\bar{u}$ and $\bar{v}$ are the maximal solutions of (7.1) and (7.2), respectively. However, we do not know whether this compact operator $T$ is continuous.

\section{Proof of Theorem 1.3}

We first prove the following

LEMMA 8.1. Let $u \in L^{1}(\Omega)$. Assume that $u / \rho_{K} \in L^{1}(\Omega)$ for some compact set $K \subset \Omega$, where $\rho_{K}(x)=d(x, K), \forall x \in \Omega$. Then,

$$
\lim _{r \rightarrow 0} \frac{1}{\left|B_{r}\right|} \int_{B_{r}(x)}|u|=0 \quad \mathcal{H}^{N-1} \text {-a.e. on } K \text {. }
$$

Proof. Let $x \in K$. For every $y \in B_{r}(x)$ we have $\rho_{K}(y) \leq r$. Thus,

$$
\frac{1}{r^{N}} \int_{B_{r}(x)}|u| \leq \frac{1}{r^{N-1}} \int_{B_{r}(x)} \frac{|u|}{\rho_{K}} .
$$

By $\left[9\right.$, Theorem 3, p.77], we have $\mathcal{H}^{N-1}(\Sigma)=0$, where

$$
\Sigma=\left\{x \in K ; \limsup _{r \rightarrow 0} \frac{1}{r^{N-1}} \int_{B_{r}(x)} \frac{|u|}{\rho_{K}}>0\right\} .
$$

Therefore

$$
\lim _{r \rightarrow 0} \frac{1}{r^{N}} \int_{B_{r}(x)}|u|=0 \quad \forall x \in K \backslash \Sigma,
$$

and the result follows.

We now present the

Proof of Theorem 1.3. Assume by contradiction that there exists some function $u \not \equiv 0$ satisfying (1.7). Applying Lemma 4.3 with $w=-u$, one deduces that $u \geq 0$ a.e. in $\Omega$. Since $\alpha<2$, by the strong maximum principle (see Lemma A.2 below), we must have $\int_{B_{1 / 2}} u>0$. Applying Proposition A.1 to suitable smooth subdomains $\omega \subset\left[x_{1}>0\right] \cap B_{1}$ and $\omega \subset\left[x_{1}<0\right] \cap B_{1}$ such that $\left[x_{1}=0\right] \cap B_{1 / 2} \subset \partial \omega$, we deduce that there exists $\varepsilon>0$ such that

$$
u(x) \geq \varepsilon\left|x_{1}\right| \quad \forall x \in B_{1 / 2} .
$$


By Lemma 8.1, we have $u=0 \mathcal{H}^{N-1}$-a.e. on $\left[x_{1}=0\right] \cap B_{1}$. Since $u$ is quasicontinuous, this implies that $u=0$ q.e. on $\left[x_{1}=0\right] \cap B_{1}$. Applying [5, Corollary 1.3], it follows from (8.2) that

$$
(\Delta u)_{\mathrm{d}} \geq \varepsilon \Delta\left|x_{1}\right|=2 \varepsilon \mathcal{H}^{N-1} \quad \text { on }\left[x_{1}=0\right] \cap B_{1 / 2},
$$

where the subscript "d" denotes the diffuse part of the measure $\Delta u$. On the other hand, by (1.7),

In particular,

$$
\Delta u \leq 0 \quad \text { on }\left[x_{1}=0\right] \cap B_{1 / 2} .
$$

$$
(\Delta u)_{\mathrm{d}} \leq 0 \quad \text { on }\left[x_{1}=0\right] \cap B_{1 / 2} .
$$

This gives a contradiction.

\section{StUdy OF A LINEAR PROBLEM}

In this section, we study the following problem

$$
\left\{\begin{aligned}
-\Delta u+\frac{u}{\left|x_{1}\right|^{\alpha}}=\mu & \text { in } B_{1} \\
u=0 & \text { on } \partial B_{1},
\end{aligned}\right.
$$

where $\alpha>0$ and $\mu$ is a diffuse measure in $B_{1}$. The existence (and nonexistence) of solutions of (9.1) is provided by the following

TheOREM 9.1. Let $\alpha>0$ and $\mu$ be a diffuse measure in $\Omega$. We have

(i) if $\alpha<1$, then (9.1) has a solution;

(ii) if $1 \leq \alpha<2$, then (9.1) has no solution if $\mu \geq 0$ and $\mu \neq 0$;

(iii) if $\alpha \geq 2$, then (9.1) has a solution if, and only if, $\mu$ does not charge the set $\left[x_{1}=0\right] \cap B_{1}$.

Proof of $(i)$. This case is already covered by Theorem 1.2 since $1 /\left|x_{1}\right|^{\alpha} \in L^{1}\left(B_{1}\right)$ if $0<\alpha<1$.

Proof of (ii). Let $\mu \geq 0$ be a diffuse measure such that (9.1) has a solution. In particular,

$$
-\int_{B_{1}} u \Delta \zeta+\int_{B_{1}} \frac{u}{\left|x_{1}\right|^{\alpha}} \zeta=\int_{B_{1}} \zeta d \mu \geq 0 \quad \forall \zeta \in C_{0}^{2}(\bar{\Omega}), \zeta \geq 0 \text { in } B_{1} .
$$

Thus, by Theorem $1.3, u=0$ a.e. We conclude that $\mu=0$.

Proof of $($ iii $) .(\Rightarrow)$ Assume that (9.1) has a solution $u$. In particular, we have

$$
-\int_{B_{1}} u \Delta \varphi+\int_{B_{1}} \frac{u}{\left|x_{1}\right|^{\alpha}} \varphi=\int_{B_{1} \backslash\left[x_{1}=0\right]} \varphi d \mu
$$

for every $\varphi \in C_{0}^{2}(\bar{\Omega})$ such that $\operatorname{supp} \varphi \cap\left[x_{1}=0\right]=\emptyset$. Given $\psi \in C_{0}^{\infty}(\mathbb{R})$ such that $\psi(t)=1$ if $|t| \leq 1 / 2$ and $\operatorname{supp} \psi \subset[-1,1]$, let

$$
\psi_{n}(x)=\psi\left(n x_{1}\right) \quad \forall x \in B_{1}, \quad \forall n \geq 1 .
$$

Since $u / x_{1}^{2} \in L^{1}\left(B_{1}\right)$, we have

$$
n^{2} \int_{\substack{B_{1} \\\left[\left|x_{1}\right| \leq \frac{1}{n}\right]}}|u| \leq \int_{B_{1}} \frac{|u|}{x_{1}^{2}} \rightarrow 0 \quad \text { as } n \rightarrow \infty .
$$


Thus,

$$
\int_{B_{1}}|u|\left|\nabla \psi_{n}\right| \leq C n \int_{\substack{B_{1} \\\left[\left|x_{1}\right| \leq \frac{1}{n}\right]}}|u| \rightarrow 0
$$

and

$$
\int_{B_{1}}|u|\left|\Delta \psi_{n}\right| \leq C n^{2} \int_{\substack{B_{1} \\\left[\left|x_{1}\right| \leq \frac{1}{n}\right]}}|u| \rightarrow 0 .
$$

Apply (9.2) with test function $\varphi=\zeta\left(1-\psi_{n}\right)$, where $\zeta \in C_{0}^{2}(\bar{\Omega})$. As $n \rightarrow \infty$, it follows from (9.3)-(9.4) and dominated convergence that

$$
-\int_{B_{1}} u \Delta \zeta+\int_{B_{1}} \frac{u}{\left|x_{1}\right|^{\alpha}} \zeta=\int_{B_{1} \backslash\left[x_{1}=0\right]} \zeta d \mu \quad \forall \zeta \in C_{0}^{2}(\bar{\Omega}) .
$$

In other words, $u$ also satisfies (9.1) with datum $\mu\left\lfloor_{B_{1} \backslash\left[x_{1}=0\right]}\right.$. Therefore,

$$
\mu\left\lfloor_{\left[x_{1}=0\right]}=0\right.
$$

and the result follows.

$(\Leftarrow)$ Let $\mu$ be a measure in $B_{1}$ which does not charge the set $\left[x_{1}=0\right] \cap B_{1}$. For every $n \geq 1$, let $u_{n}$ be the solution of

$$
\left\{\begin{aligned}
-\Delta u_{n}+\frac{u_{n}}{\left|x_{1}\right|^{\alpha}+1 / n}=\mu & & \text { in } B_{1}, \\
u=0 & & \text { on } \partial B_{1} .
\end{aligned}\right.
$$

Passing to a subsequence if necessary, we have $u_{n} \rightarrow u$ in $L^{1}\left(B_{1}\right)$. Moreover, by Lemmas 3.2 and 4.4,

$$
\frac{u_{n}}{\left|x_{1}\right|^{\alpha}+1 / n} \stackrel{*}{*} \frac{u}{\left|x_{1}\right|^{\alpha}}+\sigma \quad \text { weak }^{*} \text { in } \mathcal{M}(\Omega)
$$

for some diffuse measure $\sigma$ concentrated on the set $\left[x_{1}=0\right] \cap B_{1}$. Thus, $u$ is a solution of (9.1) with datum $\mu-\sigma$. By the implication " $\Rightarrow$ ", $\mu-\sigma$ cannot charge the set $\left[x_{1}=0\right] \cap B_{1}$. Therefore, $\sigma=0$ and $u$ is the unique solution of (9.1) associated to $\mu$. The proof of Theorem 9.1 is complete.

We conclude this section by showing the equivalence between (1.13) and (1.14):

Proposition 9.1. Let $\alpha \geq 1$ and $f \in L^{2}(\Omega)$. Then, $u \in X_{\alpha}$ satisfies

$$
\int_{B_{1}} \nabla u \cdot \nabla v+\int_{B_{1}} \frac{u}{\left|x_{1}\right|^{\alpha}} v=\int_{B_{1}} f v \quad \forall v \in X_{\alpha}
$$

if, and only if,

$$
\int_{B_{1}} \nabla u \cdot \nabla \varphi+\int_{B_{1}} \frac{u}{\left|x_{1}\right|^{\alpha}} \varphi=\int_{B_{1}} f \varphi \quad \forall \varphi \in C_{0}^{\infty}\left(B_{1}^{+} \cup B_{1}^{-}\right) .
$$

We first show the following 
Lemma 9.1. For every $v \in X_{1}$,

$$
v=0 \quad \text { in }\left[x_{1}=0\right] \cap B_{1} \text { in the sense of traces. }
$$

Hence,

$$
v \in H_{0}^{1}\left(B_{1}^{+}\right) \cup H_{0}^{1}\left(B_{1}^{-}\right)
$$

Proof. By Hölder's inequality, for every $x \in B_{1}$ and $r>0$ such that $B_{r}(x) \subset B_{1}$,

$$
0 \leq \frac{1}{\left|B_{r}\right|} \int_{B_{r}(x)}|v| \leq\left(\frac{1}{\left|B_{r}\right|} \int_{B_{r}(x)} v^{2}\right)^{1 / 2} .
$$

Applying Lemma 8.1 with function $v^{2}$ and $K=\left[x_{1}=0\right] \cap \bar{B}_{a}$, with any $a \in(0,1)$, we deduce that

$$
\lim _{r \rightarrow 0} \frac{1}{\left|B_{r}\right|} \int_{B_{r}(x)}|v|=0 \quad \mathcal{H}^{N-1} \text {-a.e. on }\left[x_{1}=0\right] \cap B_{1} .
$$

Thus, (9.8) follows. Since $v \in H_{0}^{1}\left(B_{1}\right)$, then $v$ belongs to $H_{0}^{1}\left(B_{1}^{+}\right) \cup H_{0}^{1}\left(B_{1}^{-}\right)$.

The main ingredient in the proof of Proposition 9.1 is the following

Lemma 9.2. If $\alpha \geq 1$, then $C_{0}^{\infty}\left(B_{1}^{+} \cup B_{1}^{-}\right)$is dense in $X_{\alpha}$ with respect to the norm

$$
\|v\|_{\alpha}=\|\nabla v\|_{L^{2}}+\|v\|_{L^{2}\left(\nu_{\alpha}\right)} .
$$

Proof. Let $v \in X_{\alpha}$. By the previous lemma, $v=0$ on $\left[x_{1}=0\right] \cap B_{1}$ in the sense of traces. Hence, for every $\varepsilon \in(0,1)$ we have

$$
\int_{\substack{B_{1} \\\left[\left|x_{1}\right|<\varepsilon\right]}} v^{2} \leq \varepsilon^{2} \int_{\substack{B_{1} \\\left[\left|x_{1}\right|<\varepsilon\right]}}|\nabla v|^{2} .
$$

Let $\left(v_{k}\right) \subset X_{\alpha}$ be the sequence given by

$$
v_{k}(x)=v(x) S\left(k x_{1}\right) \quad \forall x \in B_{1},
$$

where $S \in C^{\infty}(\mathbb{R})$ is such that

$$
S(t)=0 \quad \text { if }|t| \leq 1 \quad \text { and } \quad S(t)=1 \quad \text { if }|t| \geq 2 .
$$

Note that each $v_{k}$ vanishes in a neighborhood of the set $\left[x_{1}=0\right]$. We now show that

$$
v_{k} \rightarrow v \quad \text { in } X_{\alpha} .
$$

Indeed, by dominated convergence,

$$
v_{k} \rightarrow v \quad \text { in } L^{2}\left(B_{1} ; \nu_{\alpha}\right)
$$

Also notice that

$$
\left|\nabla v_{k}-\nabla v\right| \leq C|\nabla v| \chi_{\left[\left|x_{1}\right|<\frac{2}{k}\right]}+C k|v| \chi_{\left[\frac{1}{k}<\left|x_{1}\right|<\frac{2}{k}\right]} .
$$

Using (9.11), one deduces that

$$
\int_{B_{1}}\left|\nabla v_{k}-\nabla v\right|^{2} \leq C \int_{\substack{B_{1} \\\left[\left|x_{1}\right|<\frac{2}{k}\right]}}|\nabla v|^{2}
$$

Hence,

$$
\nabla v_{k} \rightarrow \nabla v \quad \text { in } L^{2}\left(B_{1}\right)
$$


Therefore, $v_{k} \rightarrow v$ in $X_{\alpha}$.

In order to conclude the proof, take $\left(w_{n}\right) \subset C_{0}^{\infty}\left(B_{1}\right)$ to be a sequence such that

$$
w_{n} \rightarrow v \quad \text { in } H_{0}^{1}\left(B_{1}\right) \text {. }
$$

In particular, for every $k \geq 1$,

$$
w_{n} S\left(k x_{1}\right) \rightarrow v S\left(k x_{1}\right) \quad \text { in } X_{\alpha} \quad \text { as } n \rightarrow \infty .
$$

Thus, for each $k \geq 1$, one can take $n_{k} \geq 1$ sufficiently large so that

$$
\left.\|\left(w_{n_{k}}-v\right) S\left(k x_{1}\right)\right] \|_{X_{\alpha}} \leq \frac{1}{k}
$$

Let

$$
u_{k}=w_{n_{k}} S\left(k x_{1}\right) \text { in } B_{1} .
$$

Then, $\left(u_{k}\right)$ is a sequence in $C_{0}^{\infty}\left(B_{1}^{+} \cup B_{1}^{-}\right)$and, by the triangle inequality,

$$
\left\|u_{k}-v\right\|_{\alpha} \leq\left\|u_{k}-v_{k}\right\|_{\alpha}+\left\|v_{k}-v\right\|_{\alpha} \leq \frac{1}{k}+\left\|v_{k}-v\right\|_{\alpha} \rightarrow 0
$$

as $k \rightarrow \infty$.

We now present the

Proof of Proposition 9.1. The implication " $\Rightarrow$ " is trivial, while the reverse implication " $\Leftarrow$ " can be easily deduced from the density of $C^{\infty}\left(B_{1}^{+} \cup B_{1}^{-}\right)$in $X_{\alpha}$.

\section{Appendix A. A Counterpart of the Hopf lemma For Weak SUPERSOLUTIONS}

In this appendix we prove the following counterpart of the Hopf lemma for the linear operator $-\Delta+b$ in the case of a (possibly) unbounded coefficient $b$ near $\partial \Omega$ :

Proposition A.1. Let $u \in L^{1}(\Omega), u \geq 0$ a.e., and $b \in L_{\mathrm{loc}}^{\infty}(\Omega)$ be such that $b u \in L^{1}(\Omega)$ and

$$
-\int_{\Omega} u \Delta \varphi+\int_{\Omega} b u \varphi \geq 0 \quad \forall \varphi \in C_{0}^{\infty}(\Omega), \varphi \geq 0 \text { in } \Omega .
$$

Assume that

$$
b \rho_{0}^{\alpha} \in L^{\infty}(\Omega) \text { for some } \alpha<2 .
$$

Then, for every $\omega \subset \subset \Omega$ there exists $C>0$ such that

$$
\underset{\Omega}{\operatorname{essinf}} \frac{u}{\rho_{0}} \geq C \int_{\omega} u \text {. }
$$

We first prove the

Lemma A.1. Let $u \in L^{1}(\Omega)$ and $f \in L^{1}\left(\Omega ; \rho_{0} d x\right)$ be such that

$$
-\int_{\Omega} u \Delta \varphi \geq \int_{\Omega} f \varphi \quad \forall \varphi \in C_{0}^{\infty}(\Omega), \varphi \geq 0 \text { in } \Omega .
$$

If $u \geq 0$ a.e., then

$$
-\int_{\Omega} u \Delta \zeta \geq \int_{\Omega} f \zeta \quad \forall \zeta \in C_{0}^{2}(\bar{\Omega}), \zeta \geq 0 \text { in } \Omega .
$$


Proof. Clearly, (A.4) still holds if $\varphi \in C^{2}(\bar{\Omega})$ and $\operatorname{supp} \varphi \subset \Omega$. Let $H: \mathbb{R} \rightarrow \mathbb{R}$ be a smooth convex function such that

$$
H(t)=0 \quad \forall t \leq 1 \quad \text { and } \quad H^{\prime}(t)=1 \quad \forall t \geq 2 .
$$

Given $\zeta \in C_{0}^{2}(\bar{\Omega}), \zeta \geq 0$ in $\Omega$, then $H(n \zeta) \in C^{2}(\bar{\Omega})$ and supp $H(n \zeta) \subset \Omega$ for every $n \geq 1$. Moreover,

$$
\Delta H(n \zeta)=n H^{\prime}(n \zeta) \Delta \zeta+n^{2} H^{\prime \prime}(n \zeta)|\nabla \zeta|^{2} \geq n H^{\prime}(n \zeta) \Delta \zeta .
$$

Applying (A.4) with test function $\varphi=H(n \zeta) / n$, we then obtain

$$
-\int_{\Omega} u H^{\prime}(n \zeta) \Delta \zeta \geq \int_{\Omega} f \frac{H(n \zeta)}{n} .
$$

As $n \rightarrow \infty$, (A.5) follows.

We shall also need the following version of the weak Harnack inequality:

LEMMA A.2. Let $u \in L^{1}(\Omega), u \geq 0$ a.e., and $b \in L_{\mathrm{loc}}^{\infty}(\Omega)$ be such that

$$
-\int_{\Omega} u \Delta \varphi+\int_{\Omega} b u \varphi \geq 0 \quad \forall \varphi \in C_{0}^{\infty}(\Omega), \varphi \geq 0 \text { in } \Omega .
$$

Then, for every $\omega \subset \subset \Omega$ there exists $C_{\omega}>0$ such that

$$
\underset{\omega}{\operatorname{essinf}} u \geq C_{\omega} \int_{\omega} u \text {. }
$$

In particular, if $u=0$ a.e. on a set of positive measure, then $u=0$ a.e. in $\Omega$.

Proof. Taking $\Omega$ smaller if necessary, we may assume that $b \in L^{\infty}(\Omega)$. We can also suppose that $\omega$ is path connected. We proceed in two steps:

Step 1. Proof of (A.7) when $u$ is smooth.

By the weak Harnack inequality (see [12, Theorem 8.18]), we have

$$
\inf _{B_{r}(x)} u \geq \frac{C}{r^{N}} \int_{B_{2 r}(x)} u
$$

for every $x \in \Omega$ with $B_{4 r}(x) \subset \Omega$; thus,

$$
\int_{B_{r}(x)} u \geq C \int_{B_{2 r}(x)} u
$$

Iterating (A.9) four times, one obtains

$$
\int_{B_{\frac{r}{5}}(x)} u \geq C \int_{B_{\frac{16 r}{5}}(x)} u
$$

for every $x \in \Omega$ with $B_{\frac{32 r}{5}}(x) \subset \Omega$.

Given $x_{0} \in \omega$ and $j \geq 1$, let $r=d(\omega, \partial \Omega) / 7$, and

$$
\mathcal{A}_{j}=\left\{\begin{array}{l|l}
x \in \Omega & \begin{array}{l}
\text { there exists } y \in \omega \text { such that } \\
d(x, y)<r \text { and } d_{\omega}\left(x_{0}, y\right)<j r
\end{array}
\end{array}\right\}
$$

where $d_{\omega}$ is the geodesic distance in $\omega$. We shall establish the following 
Claim.

$$
\int_{\mathcal{A}_{j}} u \geq C \int_{\mathcal{A}_{j-1}} u \quad \forall j \geq 1
$$

By the Vitali covering lemma, there exists a covering $\left(B_{r}\left(x_{i}\right)\right)_{i \in I}$ of the set

$$
\mathcal{E}_{j}=\left\{x \in \omega ; d_{\omega}\left(x, x_{0}\right)<j r\right\}
$$

such that $x_{i} \in \mathcal{E}_{j}, \forall i \in I$, and the balls $\left(B_{\frac{r}{5}}\left(x_{i}\right)\right)_{i \in I}$ are disjoint (all balls are defined in terms of the standard Euclidean distance in $\mathbb{R}^{N}$ ). Clearly,

$$
B_{\frac{r}{5}}\left(x_{i}\right) \subset \mathcal{A}_{j} \quad \forall i \in I .
$$

We now show that

$$
\left(B_{3 r}\left(x_{i}\right)\right)_{i \in I} \text { is a covering of } \mathcal{A}_{j+1} .
$$

Indeed, given $z \in \mathcal{A}_{j+1}$ let $y \in \omega$ be such that

$$
d(z, y)<r \quad \text { and } \quad d_{\omega}\left(x_{0}, y\right)<(j+1) r .
$$

Since $\omega$ is path connected, there exists $x \in \omega$ such that

$$
d_{\omega}(x, y)<r \quad \text { and } \quad d_{\omega}\left(x, x_{0}\right)<j r .
$$

In particular, $x \in \mathcal{E}_{j}$. Thus, there exists $i \in I$ such that $x \in B_{r}\left(x_{i}\right)$. We then have

$$
\begin{aligned}
d\left(z, x_{i}\right) & \leq d(z, y)+d(y, x)+d\left(x, x_{i}\right) \\
& \leq d(z, y)+d_{\omega}(y, x)+d\left(x, x_{i}\right)<r+r+r=3 r .
\end{aligned}
$$

Hence, $z \in B_{3 r}\left(x_{i}\right)$ and (A.14) follows.

Applying (A.10) and (A.13)-(A.14), we obtain

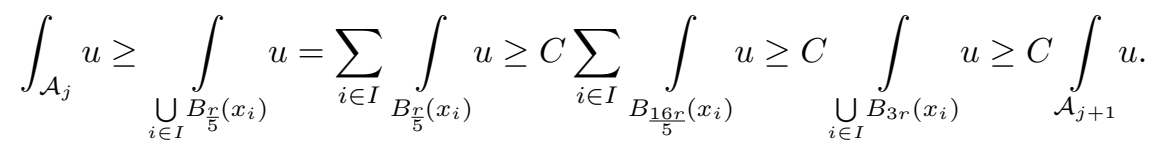

This proves (A.12).

Iterating (A.12), it follows that

$$
\int_{\mathcal{A}_{1}} u \geq C \int_{\mathcal{A}_{k}} u
$$

for some constant $C$ independent of $x_{0} \in \omega$, where $k \geq 1$ is the smallest integer such that

$$
k r \geq \sup \left\{d_{\omega}(x, y) ; x, y \in \omega\right\} .
$$

Since $\mathcal{A}_{1} \subset B_{2 r}\left(x_{0}\right)$ and $\mathcal{A}_{k} \supset \omega$, we deduce from (A.8) and (A.15) that (recall that $r=r(\omega)=d(\omega, \partial \Omega) / 7$ is fixed $)$

$$
u\left(x_{0}\right) \geq C_{\omega} \int_{\omega} u \quad \forall x_{0} \in \omega .
$$

This implies (A.7) when $u$ is smooth.

Step 2. Proof completed.

Replacing $b$ by $\|b\|_{L^{\infty}}$, we may assume that $b$ is constant. Take any domain $\widetilde{\Omega} \subset \subset \Omega$ with $\omega \subset \subset \widetilde{\Omega}$. Given $\rho \in C_{0}^{\infty}\left(B_{1}\right)$ such that $\rho \geq 0$ in $B_{1}$ and $\int_{B_{1}} \rho=1$, let 
$\rho_{\varepsilon}(x)=\frac{1}{\varepsilon^{N}} \rho\left(\frac{x}{\varepsilon}\right), \forall x \in \mathbb{R}^{N}$. Then, for $\varepsilon>0$ small, $u_{\varepsilon}=\rho_{\varepsilon} * u$ is a smooth function satisfying

$$
-\Delta u_{\varepsilon}+b u_{\varepsilon} \geq 0 \quad \text { in } \widetilde{\Omega}
$$

By the previous step,

$$
\inf _{\omega} u_{\varepsilon} \geq C \int_{\omega} u_{\varepsilon}
$$

Since

the result follows as $\varepsilon \rightarrow 0$ in (A.16).

$$
\inf _{\omega} u_{\varepsilon} \rightarrow \underset{\omega}{\operatorname{essinf}} u \quad \text { and } \quad \int_{\omega} u_{\varepsilon} \rightarrow \int_{\omega} u
$$

We now establish Proposition A.1:

Proof of Proposition A.1. Replacing $b$ by $b^{+}$if necessary, we may assume that $b \geq$ 0 . Take $\delta \in(0,1 / 2)$ small such that $\rho_{0}$ is smooth on $\bar{A}_{\delta}$ and $\omega \subset \Omega_{\delta}$, where

$$
A_{\delta}=\{x \in \Omega ; \operatorname{dist}(x, \partial \Omega)<\delta\} \quad \text { and } \quad \Omega_{\delta}=\{x \in \Omega ; \operatorname{dist}(x, \partial \Omega)>\delta\} .
$$

By Lemma A.2,

$$
\underset{\Omega_{\delta}}{\operatorname{essinf}} u \geq C \int_{\Omega_{\delta}} u \geq C \int_{\omega} u
$$

Moreover, applying Lemma A.1 we have

$$
-\int_{\Omega} u \Delta \zeta+\int_{\Omega} b u \zeta \geq 0 \quad \forall \zeta \in C_{0}^{2}(\bar{\Omega}), \zeta \geq 0 \text { in } \Omega .
$$

Given $\gamma>1$, consider

$$
v(x)=\rho_{0}(x)+\left[\rho_{0}(x)\right]^{\gamma} \quad \forall x \in A_{\delta} .
$$

A simple computation shows that $\Delta v \in L^{1}\left(A_{\delta}\right)$ and

$$
\Delta v=\left(1+\gamma \rho_{0}^{\gamma-1}\right) \Delta \rho_{0}+\gamma(\gamma-1) \rho_{0}^{\gamma-2}\left|\nabla \rho_{0}\right|^{2} \geq \frac{\gamma(\gamma-1)}{\rho_{0}^{2-\gamma}}-C_{1},
$$

since $\left|\nabla \rho_{0}\right|=1$ in $A_{\delta}$ and $\Delta \rho_{0}$ is bounded. Let $M>0$ be such that

$$
b \rho^{\alpha} \leq M \quad \text { a.e. in } \Omega \text {. }
$$

By (A.19), we have

$$
-\Delta v+\frac{M}{\rho_{0}^{\alpha}} v \leq-\frac{\gamma(\gamma-1)}{\rho_{0}^{2-\gamma}}+\frac{2 M}{\rho_{0}^{\alpha-1}}+C_{1},
$$

where we used that $v \leq 2 \rho_{0}$ since $\delta<1$. We now choose $\gamma$ so that $1<\gamma<3-\alpha$; this is possible because $\alpha<2$. Then, $2-\gamma>\alpha-1>0$. Hence, for $\delta>0$ sufficiently small (possibly depending on $\alpha$ and $M$ ) we have

$$
-\Delta v+b v \leq-\Delta v+\frac{M}{\rho_{0}^{\alpha}} v \leq 0 \quad \text { in } A_{\delta} .
$$

Let

$$
w=u-\varepsilon v \quad \text { in } A_{\delta},
$$

where $\varepsilon=C \int_{\omega} u$ and $C$ is the constant in the right-hand side of (A.17). Since $v=0$ on $\partial \Omega$, by (A.18) we get

$$
-\int_{A_{\delta}} w \Delta \psi+\int_{A_{\delta}} b w \psi \geq 0
$$


for every $\psi \in C_{0}^{2}\left(\bar{A}_{\delta}\right), \psi \geq 0$ in $A_{\delta}$, such that

$$
\operatorname{supp} \psi \subset\{x \in \bar{\Omega} ; \operatorname{dist}(x, \partial \Omega)<\delta\} .
$$

Since $v<1$ in $\bar{A}_{\delta}$, it follows from (A.17) that $w \geq 0$ a.e. in a neighborhood of $\partial A_{\delta} \cap \Omega$. Thus, by Lemma A.1 and (A.21),

$$
-\int_{A_{\delta}} w \Delta \zeta+\int_{A_{\delta}} b w \zeta \geq 0 \quad \forall \zeta \in C_{0}^{2}\left(\bar{A}_{\delta}\right), \zeta \geq 0 \text { in } A_{\delta} .
$$

Therefore, by Lemma 4.3,

$$
w \geq 0 \quad \text { a.e. in } A_{\delta} .
$$

In other words,

$$
u \geq \varepsilon v \geq \varepsilon \rho_{0} \quad \text { a.e. in } A_{\delta}
$$

which combined with (A.17) gives (A.3).

\section{Appendix B. Failure of the method of Sub AND SUPersolutions}

Throughout this appendix, we assume that $N \geq 3$. It is well-known that problem (1.4) need not have a solution if the measure $\mu$ is not diffuse. As an example, if

$$
g(x, s)=\left(s^{+}\right)^{\frac{N}{N-2}} \quad \forall(x, s) \in \Omega \times \mathbb{R},
$$

and $0 \in \Omega$, then (1.4) has no solution with datum $\mu=\delta_{0}$ (see [1]). In this case the solution of the linear problem

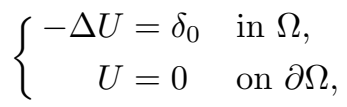

is not a supersolution of (1.4) since $g(x, U) \sim 1 /\|x\|^{N}$ is not integrable near the origin.

One may then wonder whether (1.4) has a solution under assumptions (1.5)-(1.6) if $\mu$ is not necessarily diffuse, but $\underline{U}$ and $\bar{U}$ are sub and supersolutions (i.e., if both $g(x, \underline{U})$ and $g(x, \bar{U})$ belong to $\left.L^{1}(\Omega)\right)$. This would be an extension of Proposition 5.1 for general measures $\mu$. It turns out that this is not true. In fact,

Proposition B.1. There exists a Carathéodory function $g: B_{1} \times \mathbb{R} \rightarrow \mathbb{R}$ such that (1.5)-(1.6) hold, 0 and $k /\|x\|^{N-2}$ are sub and supersolutions of

$$
\left\{\begin{aligned}
-\Delta u+g(x, u) & =\delta_{0} & & \text { in } B_{1}, \\
u & =0 & & \text { on } \partial B_{1},
\end{aligned}\right.
$$

but (B.1) has no solution.

Proof. Given $h \in C_{0}^{\infty}(\mathbb{R})$ such that $h(t) t \geq 0, \forall t \in \mathbb{R}$, and $h(1)=1$, let

$$
g(x, s)=h\left(\frac{\|x\|^{N-2}}{c_{N}} s\right) \frac{1}{\|x\|^{N}} \quad \forall(x, s) \in B_{1} \times \mathbb{R},
$$

where $1 / c_{N}=(N-2)\left|\partial B_{1}\right|$ and $\left|\partial B_{1}\right|$ is the $(N-1)$-dimensional Hausdorff measure of $\partial B_{1}$; thus,

$$
-\Delta\left(\frac{c_{N}}{\|x\|^{N-2}}\right)=\delta_{0} \quad \text { in } \mathcal{D}^{\prime}\left(\mathbb{R}^{N}\right) .
$$

The function $g$ thus defined satisfies (1.5)-(1.6). Let $M \geq 1$ be such that $\operatorname{supp} g \subset$ $[-M, M]$. Then, $v(x)=c_{N} M /\|x\|^{N-2}$ is a supersolution of (B.1) since $g(\cdot, v)=0$ and

$$
-\Delta v=M \delta_{0} \geq \delta_{0} \quad \text { in } \mathcal{D}^{\prime}\left(B_{1}\right) .
$$


Clearly, 0 is a subsolution of (1.4).

We claim that (B.1) does not have a solution. Assume by contradiction that (B.1) has a solution $u$. It is well-known that

$$
\lim _{r \rightarrow 0} \frac{1}{r} \int_{\partial B_{r}} u=(N-2) .
$$

By Proposition 4.2,

$$
0 \leq u(x) \leq \frac{c_{N}}{\|x\|^{N-2}} \quad \text { a.e. }
$$

Let $\varepsilon>0$ be such that $h(t) \geq \frac{1}{2}$ for $|t-1|<\varepsilon$. Set

$$
E_{r}=\left\{x \in \partial B_{r} ; u(x) \geq(1-\varepsilon) \frac{c_{N}}{r^{N-2}}\right\} .
$$

Thus,

$$
g(x, u(x)) \geq \frac{1}{2 r^{N}} \quad \forall x \in E_{r} .
$$

By (B.3), we have

$$
\begin{aligned}
\frac{1}{r} \int_{\partial B_{r}} u & =\frac{1}{r} \int_{E_{r}} u+\frac{1}{r} \int_{\partial B_{r} \backslash E_{r}} u \\
& \leq c_{N} \frac{\left|E_{r}\right|}{r^{N-1}}+(1-\varepsilon) c_{N} \frac{\left|\partial B_{r} \backslash E_{r}\right|}{r^{N-1}}=(N-2)-\varepsilon c_{N} \frac{\left|\partial B_{r} \backslash E_{r}\right|}{r^{N-1}} .
\end{aligned}
$$

In view of (B.2) and (B.5), we must have

$$
\lim _{r \rightarrow 0} \frac{\left|\partial B_{r} \backslash E_{r}\right|}{\left|\partial B_{r}\right|}=0 .
$$

Let $r_{0}>0$ be such that

$$
\frac{\left|E_{r}\right|}{\left|\partial B_{r}\right|} \geq \frac{1}{2} \quad \text { for every } 0<r<r_{0} .
$$

By (B.4) and (B.6), we then have

$$
\begin{aligned}
\int_{B_{1}} g(x, u) d x \geq \int_{B_{r_{0}}} g(x, u) d x & \geq \int_{0}^{r_{0}} d r \int_{E_{r}} g(r \sigma, u) d \sigma \\
& \geq \int_{0}^{r_{0}} \frac{\left|\partial B_{r}\right|}{4} \frac{d r}{r^{N}}=\frac{\left|\partial B_{1}\right|}{4} \int_{0}^{r_{0}} \frac{d r}{r}=+\infty
\end{aligned}
$$

This is a contradiction.

\section{ACKNOWLEDGMENTS}

Both authors would like to thank L. Boccardo for raising the question of existence of solutions of (1.1). They are also grateful to F. Murat for useful discussion. The second author (A.C.P.) gratefully acknowledges the invitation and the warm hospitality of the Math Department at the Università di Roma 1, where part of this work was carried out. 


\section{REFERENCES}

[1] Ph. Bénilan and H. Brezis, Nonlinear problems related to the Thomas-Fermi equation. J. Evol. Equ. 3 (2004), 673-770. Dedicated to Ph. Bénilan.

[2] L. Boccardo, T. Gallouët, and L. Orsina, Existence and uniqueness of entropy solutions for nonlinear elliptic equations with measure data. Ann. Inst. H. Poincaré Anal. Non Linéaire 13 (1996), 539-551.

[3] H. Brezis, M. Marcus, and A. C. Ponce, Nonlinear elliptic equations with measures revisited. In: Mathematical Aspects of Nonlinear Dispersive Equations (J. Bourgain, C. Kenig, and S. Klainerman, eds.), Annals of Mathematics Studies, 163, Princeton University Press, Princeton, NJ, 2007, pp. 55-110.

[4] H. Brezis and A. C. Ponce, Remarks on the strong maximum principle. Differential Integral Equations 16 (2003), 1-12.

[5] H. Brezis and A. C. Ponce, Kato's inequality when $\Delta u$ is a measure. C. R. Math. Acad. Sci. Paris, Ser. I 338 (2004), 599-604.

[6] H. Brezis and A. C. Ponce, Reduced measures for obstacle problems. Adv. Diff. Eq. 10 (2005), 1201-1234.

[7] H. Brezis and W. A. Strauss, Semilinear second-order elliptic equations in $L^{1}$. J. Math. Soc. Japan 25 (1973), 565-590.

[8] G. Dal Maso and U. Mosco, Wiener criteria and energy decay for relaxed Dirichlet problems. Arch. Rational Mech. Anal. 95 (1986), 345-387.

[9] L. C. Evans and R. F. Gariepy, Measure theory and fine properties of functions. Studies in Advanced Mathematics, CRC Press, Boca Raton, FL, 1992.

[10] D. G. de Figueiredo, Lectures on the Ekeland variational principle with applications and detours. Springer-Verlag, Berlin, 1989. Published for the Tata Institute of Fundamental Research.

[11] T. Gallouët and J.-M. Morel, Resolution of a semilinear equation in $L^{1}$. Proc. Roy. Soc. Edinburgh Sect. A 96 (1984), 275-288. Corrigenda: Proc. Roy. Soc. Edinburgh Sect. A 99 (1985), 399.

[12] D. Gilbarg and N. S. Trudinger, Elliptic partial differential equations of second order. Grundlehren der Mathematischen Wissenschaften, vol. 224, Springer-Verlag, Berlin, 1983.

[13] C.-S. Lin, A. C. Ponce, and Y. Yang, A system of elliptic equations arising in Chern-Simons field theory. J. Funct. Anal. 247 (2007), 289-350.

[14] A. Malusa and L. Orsina, Existence and regularity results for relaxed Dirichlet problems with measure data. Ann. Mat. Pura Appl. (4) $\mathbf{1 7 0}$ (1996), 57-87.

[15] M. Marcus and A. C. Ponce, Reduced limits for nonlinear equations with measures. In preparation.

[16] M. Montenegro and A. C. Ponce, The sub-supersolution method for weak solutions. Proc. Amer. Math. Soc. 136 (2008), 2429-2438.

[17] G. Stampacchia, Équations elliptiques du second ordre à coefficients discontinus. Séminaire de Mathématiques Supérieures, no. 16 (été, 1965), Les Presses de l’Université de Montréal, Montréal, Québec, 1966.

Dipartimento di MATEMATicA

Università di Roma "LA SAPIEnZA"

Piazzale A. Moro 2

00185 Roma, ItAly

DÉPARTEMENT DE MATHÉMATIQUE

Université CATHOLIQUE DE LOUVAIN

Chemin du Cyclotron 2

1348 Louvain-LA-Neuve, Belgique 